\title{
TIME LAGS AND SYNERGIES OF ONLINE ADVERTISING
}

\author{
Ralph Breuer, RWTH Aachen University, Germany \\ Malte Brettel, RWTH Aachen University, Germany
}

\begin{abstract}
With the advent of the Internet, online advertising has become a topic of increasing interest to academic research (e.g., Ha 2008; Kim and McMillan 2008). In the past decade, Internet advertising has grown beyond simple banner advertising to include new advertising models and online channels (e.g., on-demand advertising like advertisements on search engines and advertising on price-comparison websites) that make better use of the Internet's unique potential for interaction between consumer and advertiser (Rappaport 2007). However, research on the effectiveness of these online advertising channels is still rare; even though online shops are available $24 / 7$ with no more than a click between online advertisement and online shop, it remains unclear whether online advertising leads to immediate purchases or has an effect only in the long run. In addition, the synergies between the various online advertising channels are important since those channels are much closer together than are traditional ad channels (e.g., one click separates a banner ad on a web page and a search ad on a search page); ads can even appear simultaneously on a screen.
\end{abstract}

To shed light on this interesting research area, our study aims at answering the following questions:

What are the long-term and short-term effects of online advertising channels on sales?

In what way do synergies between these channels influence the effectiveness of online advertising?

We look at the sales effect of search engine marketing (SEM), banner advertising, and price-comparison advertising (PCA) using a sample of 2.8 million purchases and over 1.1 million individual costumers. The data spans a period of 365 days and was obtained from the .com-website of a leading online-platform for used and antiquarian books.

The model used to estimate carryover effects of advertising is based on the direct aggregation approach derived from Srinivasan and Weir (1988) and works as described in Herrington and Dempsey (2005). It delivers a separate $\lambda$-value for the carryover effect of each advertising channel and is complemented by a structural equation model. The estimation is done with Stata 11 using Generalized Least Squares (GLS) and is complemented by AMOS 18.

The results show that SEM has the longest carryover effect $(\lambda=0.70)$ and PCA the shortest $(\lambda=0.05)$. The $\lambda$-value of SEM corresponds to a $90 \%$ duration interval of 6.5 days; in other words, $90 \%$ of the cumulative effect of a unit impulse of SEM (banner, PCA) advertising takes place within $6.5(1.7,0.8)$ days.

Users who have been exposed to a banner ad tend to purchase the product or visit search engines or price-comparison pages. This means that the Internet user is exposed to advertising in the form of SEM or PCA after watching a banner advertisement, which is a strong indication of the existence of synergy effects. Moreover we find that SEM is almost useless without the support of other forms of online advertising since its direct sales effect is negligible compared to the synergies it facilitates.

The structural equation model used in this stud is the key element to estimate the synergies, which exist between the analyzed advertising channels. It shows that PCA has the largest total effect on sales, followed by clicks on SEM and, finally, banner clicks, which have the smallest impact. Each advertising channel has a positive total effect on sales. The importance of advertising synergies is impressive because, for banner advertising, $\sim 70 \%$ and, for SEM, $\sim 100 \%$ of the total effect can be attributed to synergies - that is, indirect effects.

All in all our research yields important insights for theory and practice since it shows how to model synergies and long-term effects of online advertising and provides meaningful insights into how to improve the allocation of advertising budgets.

References available upon request 